\title{
Expression quantitative trait loci in the developing human brain and their enrichment in neuropsychiatric disorders
}

\author{
Heath E. O'Brien ${ }^{1}$, Eilis Hannon², Matthew J. Hill ${ }^{1}$, Carolina C. Toste', Matthew J. Robertson', Joanne E. Morgan', \\ Gemma McLaughlin³, Cathryn M. Lewis ${ }^{3}$, Leonard C. Schalkwyk ${ }^{4}$, Lynsey S. Hall', Antonio F. Pardiñas', \\ Michael J. Owen ${ }^{1}$, Michael C. O'Donovan ${ }^{1}$, Jonathan Mill ${ }^{2}$ and Nicholas J. Bray ${ }^{1,5^{*}}$ (D)
}

\begin{abstract}
Background: Genetic influences on gene expression in the human fetal brain plausibly impact upon a variety of postnatal brain-related traits, including susceptibility to neuropsychiatric disorders. However, to date, there have been no studies that have mapped genome-wide expression quantitative trait loci (eQTL) specifically in the human prenatal brain.

Results: We performed deep RNA sequencing and genome-wide genotyping on a unique collection of 120 human brains from the second trimester of gestation to provide the first eQTL dataset derived exclusively from the human fetal brain. We identify high confidence cis-acting eQTL at the individual transcript as well as whole gene level, including many mapping to a common inversion polymorphism on chromosome 17q21. Fetal brain eQTL are enriched among risk variants for postnatal conditions including attention deficit hyperactivity disorder, schizophrenia, and bipolar disorder. We further identify changes in gene expression within the prenatal brain that potentially mediate risk for neuropsychiatric traits, including increased expression of C4A in association with genetic risk for schizophrenia, increased expression of $\angle R R C 57$ in association with genetic risk for bipolar disorder, and altered expression of multiple genes within the chromosome 17q21 inversion in association with variants influencing the personality trait of neuroticism.
\end{abstract}

Conclusions: We have mapped eQTL operating in the human fetal brain, providing evidence that these confer risk to certain neuropsychiatric disorders, and identifying gene expression changes that potentially mediate susceptibility to these conditions.

\section{Background}

Results from genome-wide association studies (GWAS) have established an important role for common non-coding genetic variation in complex diseases $[1,2]$. These associations are likely to reflect variants that influence gene expression, through effects on RNA transcription, splicing, or stability. By combining genome-wide genotyping with transcriptome profiling, variants

\footnotetext{
* Correspondence: BrayN3@Cardiff.ac.uk

${ }^{1} \mathrm{MRC}$ Centre for Neuropsychiatric Genetics \& Genomics, Division of Psychological Medicine \& Clinical Neurosciences, Cardiff University, Cardiff, UK

${ }^{5} \mathrm{MRC}$ Centre for Neuropsychiatric Genetics \& Genomics, Cardiff University School of Medicine, Hadyn Ellis Building, Maindy Road, Cardiff CF24 4HQ, UK Full list of author information is available at the end of the article
}

associated with altered gene expression can be mapped on a genomic scale as expression quantitative trait loci (eQTL) $[3,4]$. Although eQTL are often shared between tissues, they can also operate in a tissue-specific manner [4-6], as a result of cellular differences in the expression of the trans-regulators that interact with these loci [7], as well as in the epigenetic modification and accessibility of the regulatory elements in which they are located $[8,9]$.

Genetic influences on gene regulation that are active during human prenatal brain development plausibly impact upon a variety of postnatal, brain-related phenotypes. For example, we have previously shown that genetic variants associated with DNA methylation in the human fetal brain are enriched among variants associated with schizophrenia [10], a severe psychiatric 
disorder with a hypothesized early neurodevelopmental component [11, 12]. Common genetic variants associated with schizophrenia are also reported to be enriched within open chromatin (an index of regulatory regions) in the developing human cerebral cortex [13], and there are reports of association between gene expression in fetal brain and schizophrenia risk alleles at individual loci, e.g., $[6,14]$. However, apart from one previous investigation that included a relatively small subset of fetal brains $(n=38)$ [15], no study has explored genetic effects on gene expression in the human prenatal brain on a genome-wide scale.

In the present study, we carried out deep RNA sequencing and genome-wide genotyping on a large collection of human brains from the second trimester of gestation $(n=120)$, providing the first eQTL dataset derived exclusively from the prenatal human brain. We identify high confidence cis-acting eQTL at the individual transcript as well as whole gene level, including many mapping to a common inversion polymorphism on chromosome 17q21. We further show that fetal brain eQTL are enriched among risk variants for neuropsychiatric disorders and identify genetically influenced changes in gene expression within the developing brain that potentially mediate risk for these conditions.

\section{Results}

We performed strand-specific, whole transcriptome sequencing of total RNA derived from brain tissue from 120 human fetuses aged 12-19 post-conception weeks (PCW), generating a median 119 million read pairs per sample (Additional file 1: Table S1). Expression measures were derived for 144,448 Ensembl transcripts, annotated to 28,875 genes. Genomic DNA from each sample was genotyped for approximately 710,000 single nucleotide polymorphisms (SNPs), followed by genotype imputation using the Haplotype Reference Consortium r1.1 panel [16]. After strict quality control, 5.8 million SNPs were retained for analysis. Consistent with the GTEx Consortium [4], we controlled gene expression measures for latent factors using probabilistic estimation of expression residuals (PEER) [17] in addition to specified covariates and principal components from the genotyping matrix (Additional file 2: Figure S1 and Figure S2). Cis-eQTL were identified by linear regression of allele dosage against adjusted, quantile-normalized gene expression measures using FastQTL [18].

\section{Characterization of cis-eQTL in the human fetal brain}

We identified cis-eQTL (defined as variants within $1 \mathrm{Mb}$ of the transcription start site [TSS] of the regulated gene) associated with the fetal brain expression of 1329 genes (eGenes) and 3252 individual transcripts (eTranscripts) at a false discovery rate $($ FDR) $<0.05$ (Additional file 1: Table
S2 and Table S3). The majority (86\%) of eQTL detected at the whole gene level were also detected at the individual transcript level. Consistent with previous eQTL mapping studies $[5,15,19]$, significant eQTL were concentrated in proximity to the TSS of the regulated transcript (Fig. 1a). We further tested for enrichment of high confidence cis-eQTLs (FDR $<0.05)$ within functional annotations provided by the ENCODE [8] and Roadmap Epigenomics Consortium [9] projects (Additional file 1: Table S4 and Table S5). Fetal brain cis-eQTL were significantly enriched within regions annotated as TSS, flanking promoters, enhancers, weak enhancers, and CTCF binding sites, but significantly depleted in repressed regions (Fig. 1b). Identified cis-eQTL were also notably enriched within regions containing histone modifications indicative of regulatory activity (Additional file 2: Figure S3). We additionally tested for enrichment, among our high confidence cis-eQTL, of variants that we have previously found to be associated with altered DNA methylation (mQTL) in the human fetal brain [10]. Consistent with an association between DNA methylation and gene expression [20, 21], fetal brain $\mathrm{mQTL}$ were enriched sixfold among fetal brain cis-eQTL $\left(P=3.13 \times 10^{-19}\right)$.

\section{eQTL at a common inversion polymorphism on chromosome 17q21}

Twenty-one of the cis-eQTL eGenes identified in fetal brain are located within a region of chromosome $17 q 21.31$ containing a common $900-\mathrm{kb}$ inversion polymorphism and several common duplications [22, 23]. Variants within this inversion (denoted "H2"), which appears to be under positive selection in Europeans [22], have recently been implicated in neuroticism [24-26], while markers on the non-inverted form (denoted "H1") are associated with several neurodegenerative diseases [27-29]. For 13 of the eGenes in this region (e.g., KANSL1, LRRC37A, DND1P1), cis-eQTL could be largely explained by inversion status (r2 with $\mathrm{H} 1 / \mathrm{H} 2$ tag SNP rs1800547 > 0.9), with variants tagging the inverted $\mathrm{H} 2$ form associated with both increases and decreases in gene expression (Table 1). For other genes in the region (e.g., NSF, ARL17A, ARL17B), identified cis-eQTL might index duplicated expressed gene sequence as well as regulatory variants that have arisen independently on the $\mathrm{H} 1$ or $\mathrm{H} 2$ haplotypes.

\section{Tissue-sharing and evidence for fetal-specific eQTL}

We next explored the extent to which cis-eQTL identified in the fetal brain are shared among 48 adult human tissues analyzed through the Genotype-Tissue Expression (GTEx) project [4]. For this, we restricted our analyses to the eQTL we identified for fetal eGenes as the GTEx project does not currently provide eQTL data at the individual transcript level. Of the 1151 fetal brain 


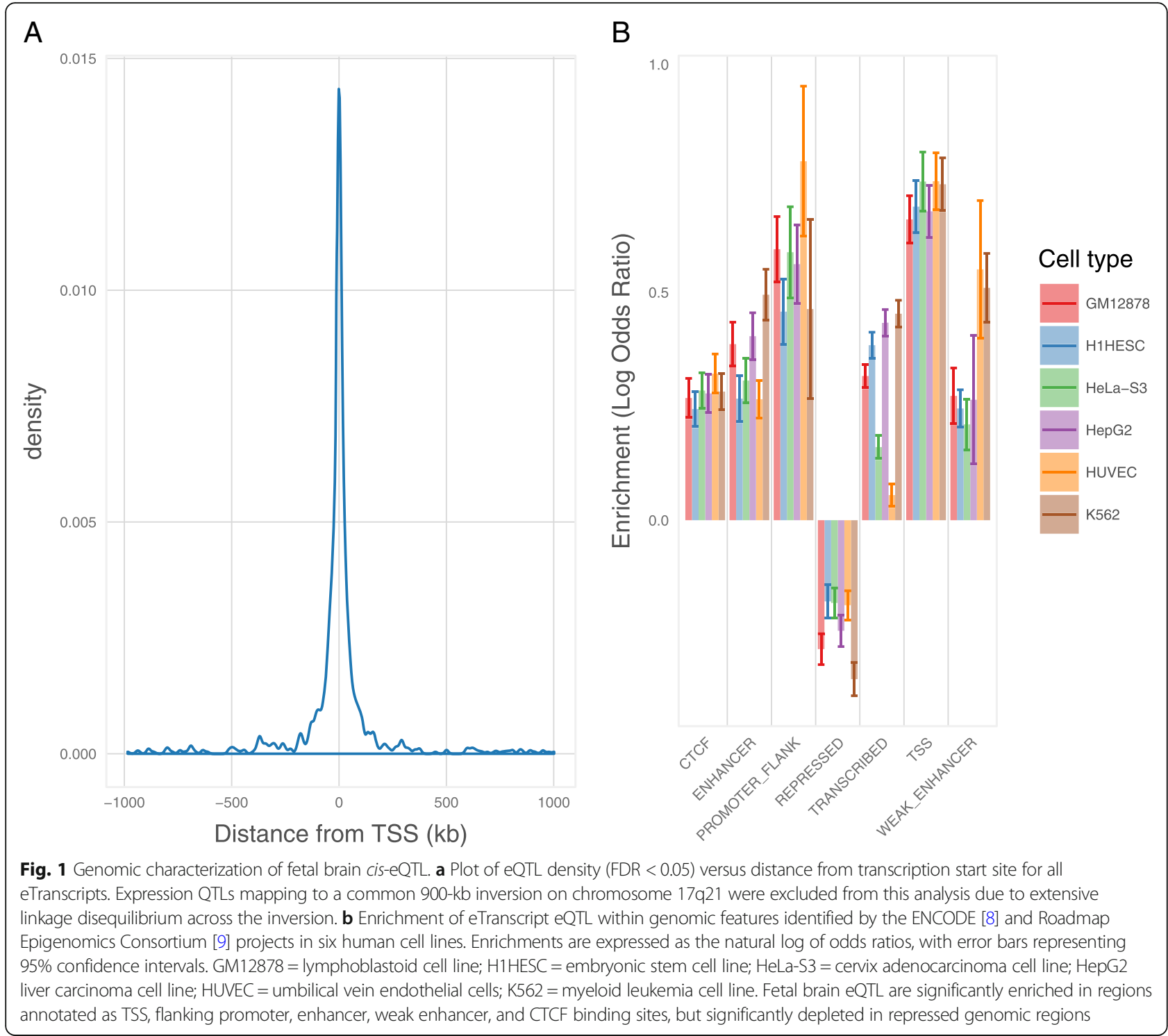

eGenes for which GTEx Consortium data were available, 979 had a top eQTL (the most significant fetal brain eQTL for that gene that was genotyped in the GTEx study) that was also observed in at least one adult tissue (at FDR < 0.05). The shared eQTL for 974 of these fetal eGenes were predicted to be active in at least two adult tissues. Tissue-sharing of fetal brain eQTL was most pronounced within regions of the adult brain, where an average of $79 \%$ of the tested fetal brain eQTL were predicted to be shared, and lowest for whole blood, where only $41 \%$ of the tested fetal brain eQTL were predicted to be shared (Fig. 2). Predicted tissue sharing was supported by estimates of $\pi_{1}$ (the proportion of true positives [30]) among fetal eQTL when tested for replication in individual GTEx tissues, with high $\pi_{1}$ estimates in adult brain regions despite relatively small GTEx sample sizes (Additional file 2: Figure S4). For 172 eGenes assayed by the GTEx Consortium, the eQTL identified in fetal brain were not associated with significant (FDR $<0.05$ ) effects on their expression in any sampled adult tissue, and these might therefore constitute fetal-specific eQTL (Additional file 1: Table S6).

\section{Regional and developmental expression of fetal eGenes in the human brain}

We used RNA sequencing data from dissected regions of the human pre- and post-natal brain, available through the BrainSpan project, to explore the spatiotemporal expression of the fetal eGenes we identified. In general, fetal eGenes were expressed fairly uniformly across dissected regions of both the fetal and adult brain, with some genes showing very high expression and others very low (Additional file 2: Figure S5 and Figure S6). Similarly, the majority of fetal eGenes displayed a reasonably stable 
Table 1 Fetal brain eGenes located at a common inversion polymorphism on chromosome 17q21

\begin{tabular}{|c|c|c|c|c|c|c|}
\hline eGene & ENSEMBL ID & Number eQTL SNPs FDR < 0.05 & Top eQTL & $\begin{array}{l}\text { Top eQTL } \\
\text { FDR }\end{array}$ & $r^{2}$ with inversion* & $\mathrm{H} 1 / \mathrm{H} 2$ expression ratio \\
\hline NSF & ENSG00000073969 & 40 & rs3874943 & 4.05E-06 & 0.14 & 1.02 \\
\hline KANSL1 & ENSG00000120071 & 2226 & rs2668690 & 2.81E-14 & 0.94 & 0.89 \\
\hline LRRC37A & ENSG00000176681 & 2225 & rs3785884 & 5.89E-17 & 0.97 & 0.80 \\
\hline ARL17A & ENSG00000185829 & 34 & rs1863115 & 7.03E-04 & 0.11 & 1.00 \\
\hline KANSL1-AS1 & ENSG00000214401 & 2215 & rs62057151 & $1.72 \mathrm{E}-11$ & 0.94 & 0.81 \\
\hline LRRC37A4P & ENSG00000214425 & 2210 & rs55944735 & $7.40 \mathrm{E}-12$ & 0.97 & 1.96 \\
\hline$A R L 17 B$ & ENSG00000228696 & 48 & rs1863115 & $1.82 \mathrm{E}-06$ & 0.11 & 1.02 \\
\hline LRRC37A2 & ENSG00000238083 & 2213 & rs753236 & 7.11E-15 & 0.94 & 0.83 \\
\hline NSFP1 & ENSG00000260075 & 54 & rs1863115 & $2.89 E-14$ & 0.11 & 1.22 \\
\hline AC005670.2 & ENSG00000262633 & 1036 & rs2696689 & 4.02E-03 & 0.65 & 0.91 \\
\hline MAPK8IP1P2 & ENSG00000263503 & 2276 & rs111447859 & $1.50 E-23$ & 0.97 & 0.39 \\
\hline DND1P1 & ENSG00000264070 & 2199 & $17: 44219831$ & $1.73 \mathrm{E}-08$ & 0.97 & 1.38 \\
\hline MAPT-ASI & ENSG00000264589 & 1816 & rs60814418 & $3.01 \mathrm{E}-02$ & 0.61 & 1.15 \\
\hline RN7SL199P & ENSG00000265315 & 2203 & rs56180212 & 2.47E-09 & 0.97 & 0.78 \\
\hline RN7SL656P & ENSG00000265411 & 2204 & rs56180212 & 2.17E-09 & 0.97 & 0.78 \\
\hline AC138645.1 & ENSG00000266497 & 2218 & rs4327090 & $5.75 \mathrm{E}-11$ & 0.94 & 0.82 \\
\hline AC091132.3 & ENSG00000266504 & 2213 & rs4327090 & $5.12 \mathrm{E}-11$ & 0.94 & 0.82 \\
\hline AC091132.4 & ENSG00000266918 & 2066 & rs9899833 & $1.55 E-02$ & 0.54 & 1.06 \\
\hline AC091132.5 & ENSG00000267198 & 39 & rs79475968 & 7.94E-05 & 0.04 & 1.03 \\
\hline AC091132.6 & ENSG00000267246 & 2253 & rs753236 & 7.61E-11 & 0.94 & 0.76 \\
\hline Metazoa_SRP & ENSG00000278770 & 2215 & rs4327090 & $2.30 \mathrm{E}-09$ & 0.94 & 1.54 \\
\hline
\end{tabular}

*Consistent with Stefansson et al [22], inversion status was inferred through genotype at SNP rs1800547, with the MAPT haplotype "H2" used to denote the inversion and " $\mathrm{H} 1$ " the non-inverted form

pattern of expression across brain development, even when the associated eQTLs were predicted to be fetal-specific (Additional file 2: Figure S7). A notable exception to this pattern was observed in the developmental expression of the predicted fetal-specific eGene HBG1, encoding hemoglobin subunit gamma 1 (a component of fetal hemoglobin), which showed the expected preponderance in prenatal brain (Additional file 2: Figure S8).

\section{Enrichment of fetal brain cis-eQTL within genetic variants associated with neuropsychiatric traits}

Genetic effects on gene expression operating in the human fetal brain could influence a variety of post-natal, brain-related phenotypes. We tested for enrichment among high confidence fetal brain eQTL (FDR < 0.05 ) of variants associated with seven neuropsychiatric traits (attention deficit hyperactivity disorder, anorexia nervosa, autism spectrum disorder, bipolar disorder, major depressive disorder, neuroticism, and schizophrenia) using large-scale GWAS data [26, 31-36]. For these analyses, we focused on transcript-level eQTL as these largely encompass those identified at the gene level and are likely to additionally index subtle regulatory variation (e.g., those affecting splicing, or transcript-specific promoters/ enhancers) that could be relevant to complex traits [37]. We assessed enrichment among fetal eQTL of trait-associated variants at four GWAS $P$ value thresholds for each trait $\left(P<5 \times 10^{-5}, 5 \times 10^{-6}, 5 \times 10^{-7}\right.$, and $5 \times 10^{-8}$ ), using a method that accounts for allele frequency, linkage disequilibrium, and local gene density [38]. As a comparison, we performed identical analyses on similar sized GWAS data for five non-brain traits (body mass index, coronary artery disease, inflammatory bowel disease, height, and type 2 diabetes) [39-43]. Fetal brain transcript eQTL were notably enriched $(P<0.001)$ within trait-associated variants for three neuropsychiatric disorders (attention deficit hyperactivity disorder, bipolar disorder, and schizophrenia) and one non-brain trait (inflammatory bowel disease) at one or more GWAS $P$ value threshold (Fig. 3; Additional file 1: Table S7).

\section{Identification of gene expression changes in fetal brain associated with neuropsychiatric traits}

Given evidence for a potential role of fetal brain eQTL in neuropsychiatric traits, we next applied summary data-based Mendelian randomization (SMR) to identify genetically influenced changes in gene expression in the fetal brain that could mediate genetic risk for these 


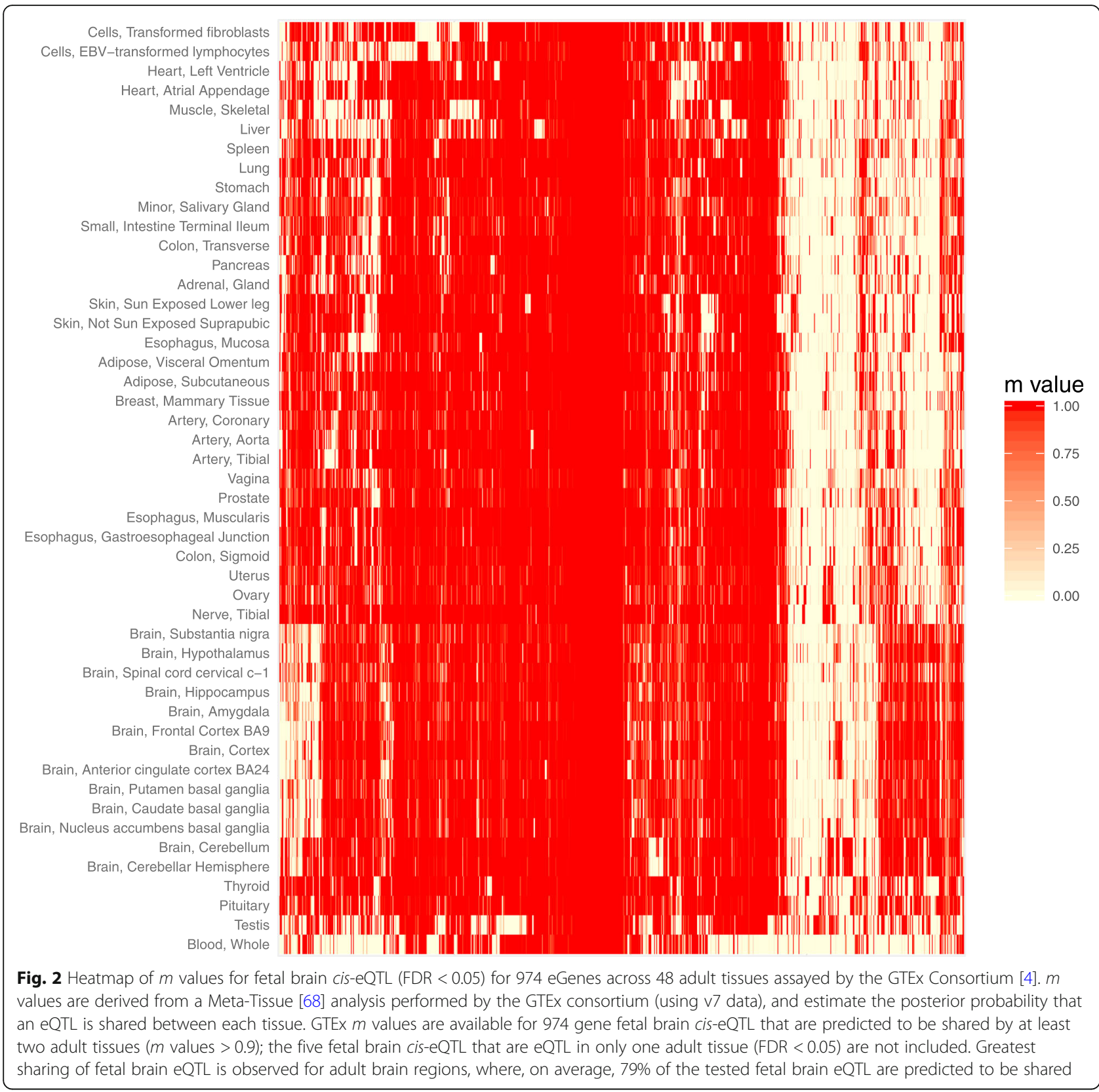

conditions. This approach employs Mendelian randomization to test for pleiotropic associations between gene expression and complex traits using eQTL and trait GWAS summary data [44, 45]. We tested for joint associations between gene expression and risk for the seven neuropsychiatric traits (attention deficit hyperactivity disorder, anorexia nervosa, autism spectrum disorder, bipolar disorder, major depressive disorder, neuroticism, and schizophrenia) using the same GWAS datasets as we used for enrichment analyses [26, 31-36]. We considered only eGenes and eTranscripts for which fetal brain eQTL were identified at FDR $<0.05$, and report associations that survive correction for multiple tests (eGenes, $P$-SMR $=3.76 \times 10^{-5}$; eTranscripts, $P$-SMR $\left.<1.54 \times 10^{-5}\right)$. In addition, we only report associations that are non-significant $(P>0.05)$ for the HEIDI (heterogeneity in dependent instruments) test, indicating that they are unlikely to be driven by linkage between the eQTL and independent risk variants [44]. In total, we identified 15 eGenes and 34 eTranscripts where expression in fetal brain was pleiotropically associated with at least one neuropsychiatric trait and conformed to the above criteria (Additional file 1: Table S8 and Table S9, respectively).

We identified 6 eGenes and 14 eTranscripts for which fetal brain expression was associated with schizophrenia risk. These include reduced expression of $A B C B 9$, 


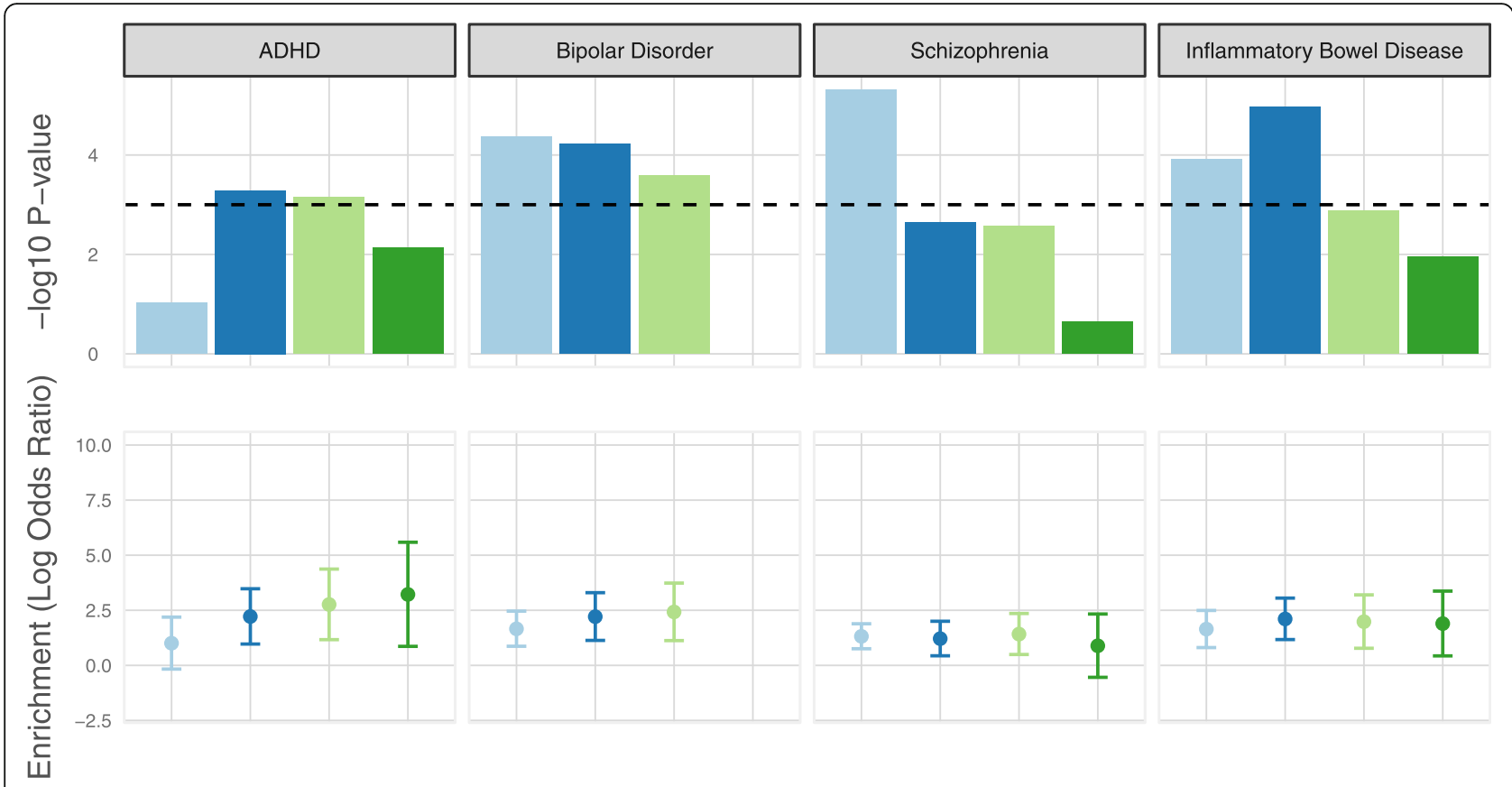

GWAS P-value Threshold $-\odot-5 e-05-\bullet-5 e-06-0-5 e-07-\bullet-5 e-08$

Fig. 3 Enrichment of fetal brain transcript eQTL within variants associated with complex post-natal traits. Enrichments were tested at four GWAS $P$ value thresholds $\left(P<5 \times 10^{-5}, 5 \times 10^{-6}, 5 \times 10^{-7}\right.$, and $\left.5 \times 10^{-8}\right)$ for each trait. Top panel shows $\log 10 P$ values at each threshold. The bottom panel shows the natural log of the odds ratio, with $95 \%$ confidence intervals, at each threshold. Only traits for which the significance of eQTL enrichment survives correction for multiple tests $(P<0.001$; dotted line in upper panel) at one or more GWAS $P$ value threshold are shown. There are no overlaps between fetal brain eQTL and risk variants for bipolar disorder at the $P<5 \times 10^{-8}$ threshold

encoding ATP Binding Cassette Subfamily B Member 9 $\left(P\right.$-SMR $\left.=2.11 \times 10^{-5}\right)$, which has been previously implicated through SMR studies of schizophrenia GWAS in blood [44, 46] and other non-brain adult tissues [45], and increased expression of CSPG4P11, encoding Chondroitin Sulfate Proteoglycan 4 Pseudogene 11 (P-SMR = $\left.6.66 \times 10^{-6}\right)$, which has also been reported in an SMR study of adult human brain gene expression data [45]. We also observed association between schizophrenia and reduced fetal brain expression of a transcript of KLC1, encoding Kinesin Light Chain 1 ( $P$-SMR $=2.95 \times$ $10^{-7}$ ), which has recently been implicated in schizophrenia risk through a splicing QTL in the adult brain [47]. The most significant association with schizophrenia $\left(P\right.$-SMR $\left.=6.3 \times 10^{-8}\right)$ was in the expression of $C 4 A$, encoding Complement $C 4 A$, which exhibited increased expression in association with risk variation (Fig. 4). Genetic association between schizophrenia and the major histocompatibility complex (MHC) locus on chromosome 6 has been shown to partly reflect common gene copy number variants resulting in increased expression of C4A in the adult human brain [48]. The present data suggest that pathogenic effects of variation at the C4 locus might begin in utero. We also observed several novel associations with schizophrenia, including reduced expression of transcripts of FLOT1, encoding
Flotillin $1\left(P\right.$-SMR $\left.=5.0 \times 10^{-7}\right)$, increased expression of a transcript of MSANTD2, encoding Myb/SANT DNA Binding Domain Containing $2\left(P\right.$-SMR $\left.=2.8 \times 10^{-6}\right)$, and increased expression of HIST1H4L, encoding Histone Cluster 1 H4 Family Member L $\left(P\right.$-SMR $\left.=4.4 \times 10^{-7}\right)$.

We observed association between variants influencing the personality trait of neuroticism and the expression of 7 eGenes and 18 eTranscripts in fetal brain. The majority of these (4 eGenes and 17 eTranscripts) were located at the common inversion polymorphism on chromosome 17q21. The most significant SMR associations were observed for transcripts of LRRC37A, encoding Leucine Rich Repeat Containing 37A (smallest $P$-SMR $=2.33 \times 10^{-13}$ ) and KANSL1, encoding KAT8 Regulatory NSL Complex Subunit 1 (smallest $P$-SMR $=8.1 \times 10^{-12}$ ), with variants conferring higher neuroticism scores associated with increased expression of both genes. The three neuroticism-associated genes that did not map to the inversion are functionally uncharacterized, but for one of these genes (AC022784.7), the cis-eQTL is predicted to be fetal-specific. The only neuroticism-associated transcript that did not map to chromosome 17q21 is annotated to the ORC4 gene, encoding Origin Recognition Complex Subunit 4 $(P-\mathrm{SMR}=$ $1.91 \times 10^{-7}$ ).

Reduced expression of one transcript, annotated to the RPL31P12 gene (encoding Ribosomal Protein L31 

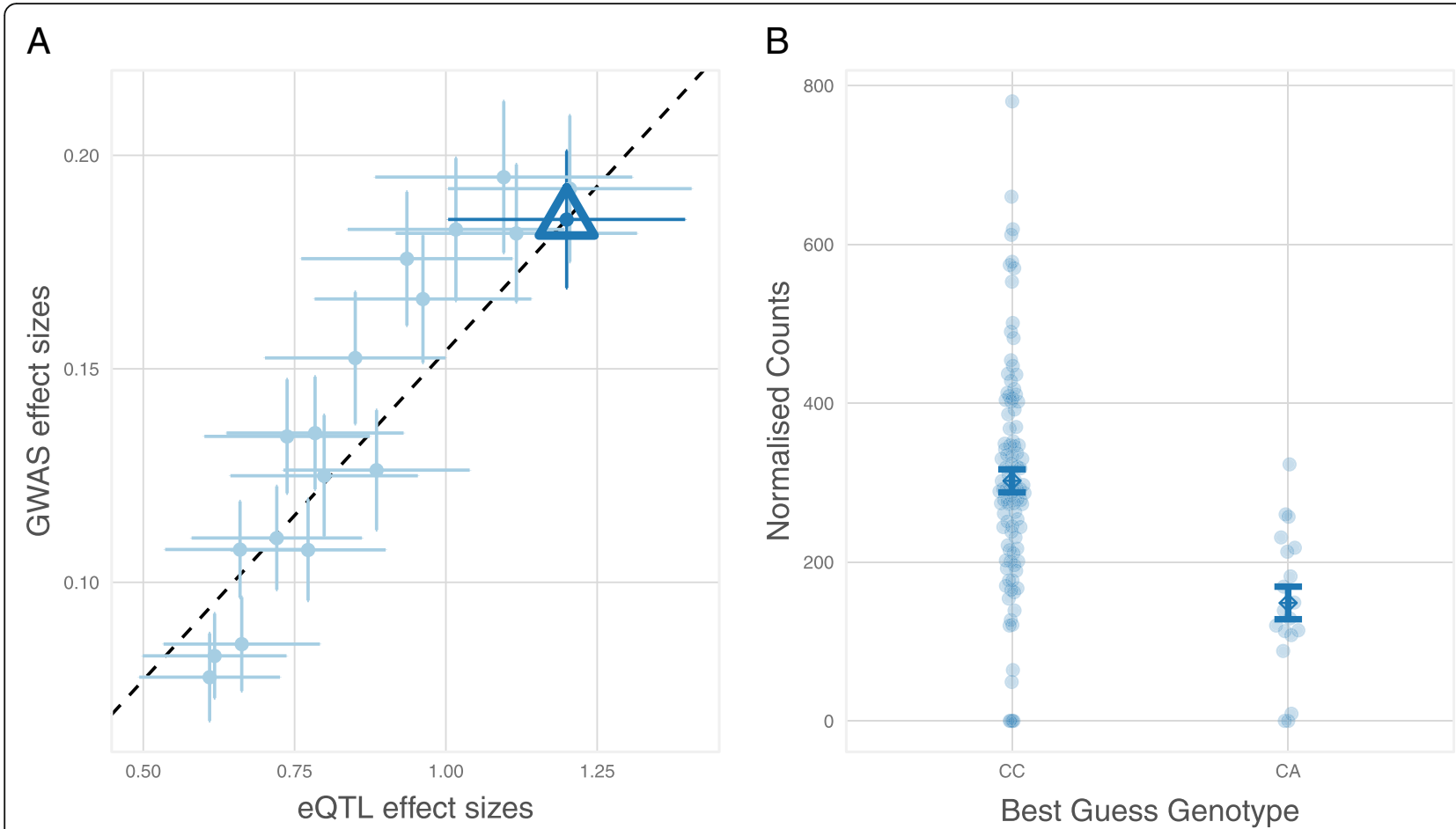

Fig. 4 Association between expression of the Complement C4A gene in fetal brain and genetic risk for schizophrenia. a Effect sizes of SNPs from schizophrenia GWAS [36] plotted against their effect sizes as fetal brain cis-eQTL for C4A. The triangular data point indicates the variant with the lowest P-SMR. Error bars are the standard errors of SNP effects. $\mathbf{b}$ Homozygotes for the schizophrenia-associated C-allele of rs9267544 exhibit increased expression of C4A in fetal brain compared with heterozygotes for this SNP. Error bars represent standard errors

Pseudogene 12), was associated with major depressive disorder $\left(P\right.$-SMR $\left.=2.19 \times 10^{-6}\right)$, while increased expression of the RPS26 gene (encoding Ribosomal Protein $S 26)$ was associated with genetic risk for anorexia nervosa $\left(P-S M R=2.34 \times 10^{-6}\right)$. Increased expression of the LRRC57 gene, encoding Leucine Rich Repeat Containing 57 , was associated with bipolar disorder (P-SMR = $\left.3.07 \times 10^{-5}\right)$. In addition, a transcript annotated to GNL3, encoding G Protein Nucleolar 3, was associated with both bipolar disorder $\left(P-S M R=2.17 \times 10^{-6}\right)$ and schizophrenia $\left(P-\mathrm{SMR}=2.71 \times 10^{-7}\right)$, with risk alleles for both disorders associated with increased expression of this transcript in fetal brain.

We additionally explored whether eQTLs identified in the adult human brain (dorsolateral prefrontal cortex) [49] that have been implicated in neuropsychiatric traits in a previous SMR study [45] are potentially also operating in the fetal brain. For four fetal eGenes, we observed strong linkage disequilibrium in our samples $\left(r^{2}>0.8\right)$ between the most significant fetal brain eQTL and the adult brain eQTL implicated in schizophrenia risk through SMR (Additional file 1: Table S10). In addition to CSPG4P11, which showed a significant SMR association with schizophrenia in the present study, these included CD46, encoding the CD46 complement regulatory protein, GOLGA2P7, encoding GOLGA2 pseudogene 2, and SLCO4C1, encoding Solute Carrier Organic Anion Transporter Family Member 4C1. There was no apparent overlap between adult brain eQTL implicated in neuroticism and eQTL regulating the fetal eGenes identified in this study.

\section{Discussion}

We present the first genome-wide eQTL mapping study performed exclusively on the prenatal human brain. Through deep RNA sequencing, we have been able to interrogate eQTL at the individual transcript as well as whole gene level, providing a more precise elucidation of common genetic influences on gene expression in the developing brain and the molecular differences that could confer susceptibility to neuropsychiatric disorders.

Using a method that controls for the number of SNPs tested at each locus as well as the number of genes/transcripts assayed [4, 18], we identified high confidence cis-eQTL regulating 1329 genes and 3252 individual transcripts in the human fetal brain. This approximates the number of eGenes identified using the same procedure in adult brain collections of comparable size; for example, in 92 samples from the adult frontal cortex, the GTEx Consortium identified cis-eQTL (FDR < 0.05) for 1588 genes [4]. However, this is likely to constitute only a small percentage of the genes expressed in fetal brain 
that are subject to variable genetic cis-regulation. Studies in adult human tissues have indicated that a sizeable proportion of expressed genes are variably cis-regulated $[4,50,51]$, with eQTL mapping studies showing the expected strong correlation between eQTL discovery and sample size [4]. Larger collections of fetal brain are therefore likely to yield many additional eQTL, with resulting improvements in power to identify gene expression changes relevant to brain-related traits.

Many of the fetal brain eQTL we identified map to a common $900-\mathrm{kb}$ inversion polymorphism on chromosome 17q21.31. The eQTL for 13 of the genes in the region are largely tagged by the inversion, which was associated with both increases and decreases in gene expression. Although our analyses of tissue-sharing suggest that none of the eQTL mapping to this region are specific to the fetal brain, only one study to our knowledge has specifically reported any of the gene expression differences we observe [52]. This previous study reported differences between $\mathrm{H} 1$ and $\mathrm{H} 2$ in the expression of six genes in the adult human brain, which, congruent with our data, included increased expression of $L R R C 37 A$ in association with the inverted $\mathrm{H} 2$ form.

Of the tested neuropsychiatric traits, we observed notable enrichment among high confidence fetal brain eQTL of variants associated with attention deficit hyperactivity disorder (ADHD), bipolar disorder, and schizophrenia. We also observed nominally significant enrichment of variants associated with autism at lower GWAS $P$ value thresholds (Additional file 1: Table S7), although we note that the number of tested variants for this was smaller than for other brain-related traits, limiting statistical power. Like autism, ADHD is considered to be a neurodevelopmental condition, but the genes involved and the developmental timing of their action are largely unknown. We observed an average 13-fold enrichment of ADHD-associated variants within fetal brain eQTL across the four GWAS $P$ value thresholds tested, suggesting an important prenatal genetic component to this condition. Our finding of an enrichment of schizophrenia-associated variants among fetal brain eQTL is also consistent with the long hypothesized early neurodevelopmental component to this disorder $[11,12]$. Although less commonly conceptualized as neurodevelopmental in origin, we observed a similar enrichment of risk variants for bipolar disorder among high confidence fetal brain eQTL. In contrast, we observed no enrichment of risk variants for major depressive disorder, despite similar numbers of tested risk-associated variants at each GWAS $P$ value threshold (Additional file 1: Table S7). Among non-brain traits, the most pronounced enrichment of fetal brain eQTL was observed for variants associated with inflammatory bowel disease (average sevenfold enrichment across the four GWAS $P$ value thresholds), suggesting a developmental component to this condition.
The eQTL giving rise to the identified pleiotropic associations between gene expression in the fetal brain and risk for neuropsychiatric disorders do not, for the most part, appear to be fetal-specific. However, their activity within the brain at this timepoint makes it plausible that their pathogenic effects start prenatally, even if they continue into adulthood. Indeed, several of the genes that we implicate are known to have important roles in early brain development. For example, leucine-rich repeat containing genes (which include LRRC37A, implicated in neuroticism, and LRRC57, implicated in bipolar disorder) are known to be key organizers of excitatory and inhibitory synapse formation [53]. Similarly, FLOT1, which exhibited reduced expression in association with genetic risk for schizophrenia, has been found to promote the formation of hippocampal glutamatergic synapses [54], which appear to be decreased in the disorder [55]. Increased expression of $C 4 A$ in association with schizophrenia risk variation was originally reported in the adult brain [48], where the authors provided data to support a role for this gene in synaptic pruning. Although this process is important for maturation of regions such as the frontal cortex in adolescence, it begins (and is for some brain regions pronounced) during the perinatal period [56]. Our findings thus suggest that as well as influencing synaptic pruning during adolescence, effects of $C 4$ risk variation could initially impact neural connectivity at this early phase of synaptic refinement.

\section{Conclusion}

We have mapped high confidence eQTL operating in the human fetal brain. Although a large proportion of these continue to operate in adulthood, their activity at this timepoint and enrichment among risk variants for certain neuropsychiatric disorders suggest that their influence on brain-related traits may at least start at this early stage of development. We identify several genes and individual transcripts for which expression in the fetal brain could mediate genetic risk for neuropsychiatric traits, and these might therefore warrant further neurobiological investigation. We have made the summary eQTL data available [57] so that others can explore neurodevelopmental antecedents to these and other brain-related conditions.

\section{Methods \\ Samples}

Human fetal brain tissue from elective terminations of pregnancy $(12-19 \mathrm{PCW})$ was provided by the Human Developmental Biology Resource (HDBR) (http:// www.hdbr.org). Fetal age was determined by the HDBR through foot length and knee-to-heel length measurements. Fetal sex was determined by karyotyping and confirmed in this study through male-specific expression 
of genes on the Y-chromosome and heterozygosity for $\mathrm{X}$-chromosome markers in females. No other phenotypic or demographic information was available. Brain tissue was obtained frozen and had not been dissected into regions. Total RNA was extracted from half of the available brain tissue from each fetus using Tri-Reagent (Thermo Fisher Scientific), while the other half of each sample was processed for genomic DNA using standard phenol-chloroform extraction.

\section{Genotyping}

Genomic DNA from each sample was genotyped for approximately 710,000 SNPs using the Infinium OmniExpress-24 BeadChip array (Illumina). Genotyping data was subjected to stringent quality control using PLINK v1.9. and the check-bim utility from http:// www.well.ox.ac.uk/ wrayner/tools/index.html. Samples with $>5 \%$ missing markers, ambiguous sex assignments, or anomalous heterozygosity were excluded. SNPs that were missing in $>5 \%$ of samples or had minor allele frequency less than 0.01 were removed, as were $\mathrm{A} / \mathrm{T}$ and G/C SNPs with minor allele frequencies $>0.4$. The SNP strand and ref/alt assignment were updated to match the Human Reference Consortium (HRC) version 1.1, and SNPs where the minor allele frequency differed by $>0.2$ from the HRC were removed. Additional genotypes were imputed from the HRC panel using minimac3 and Eagle v2.3 phasing through the Michigan Imputation Server (https://imputationserver.sph.umich.edu/index.html).

SNPs were annotated with rsID numbers from dbSNP v149 and converted to GRCh38 coordinates using CrossMap [58] with chain files from the UCSC Genome Browser (https://genome.ucsc.edu/). Non-SNP positions and duplicated sites identified by checkVCF (https:// github.com/zhanxw/checkVCF) were filtered out, as were sites with an imputation $r^{2}$ less than 0.8 , minor allele frequency less than 0.05, or Hardy-Weinberg Equilibrium violation $P$ values less than $1 \times 10^{-4}$. Genotypes from DNA were compared with those from RNA sequencing to confirm matching of genotype and gene expression measures for each sample.

\section{RNA sequencing library preparation}

Total RNA was treated with TURBO DNase (Thermo Fisher Scientific) and purified using the RNeasy Micro kit (Qiagen). Integrity of RNA was assessed using the Agilent 2100 Bioanalyzer. RNA-Seq libraries were prepared using $1 \mu \mathrm{g}$ of purified total RNA and the TruSeq Stranded Total RNA Library Prep kit (Illumina), depleting ribosomal RNA using the Ribo-Zero Gold reagent (Illumina), and modifying the RNA fragmentation times for lower RIN samples $(<7)$ according to manufacturer instructions. Library size was assessed using High Sensitivity DNA Analysis kits (Agilent) on the Agilent 2100
Bioanalyzer and libraries quantified using KAPA Library Quantification Kits (KAPA Biosystems) prior to pooling. Libraries were sequenced on the Illumina HiSeq 2500 or HiSeq 4000 systems, generating at least 50 million read pairs (100 million reads) per sample.

\section{Gene expression analyses}

Sequencing adapters were removed from reads with Cutadapt using the Trim Galore! Wrapper script. QC analyses were carried out using FastQC before and after adapter trimming, and additionally using RSeQC [59] after mapping to the GRCh38 human genome reference sequence with HISAT2 [60]. We used bcftools v1.6 to call SNPs in the mapping files and compared them to the imputed genotypes using bcftools gtcheck. Transcript abundance was quantified by pseudoalignment of sequencing reads to transcript sequences derived from the GRCh38 human genome reference sequence and Ensembl (version 81) reference annotation using Kallisto [61]. Reads were aggregated at the gene level using tximport [62] and biomaRt [63]. Between-sample normalization and variance-stabilizing transformation were carried out using DESeq2 [64], and genes/transcripts that had VST-normalized count values $>5$ in 10 or more samples were included in all subsequent analyses. The expression matrix was quantile-normalized before and after correcting for covariates (see below), and principal component analysis was used to assess between-sample heterogeneity (Additional file 2: Figure S1). The influence of covariates on gene expression was also quantified using the variancePartition package in $R$ [65] (Additional file 2: Figure S2).

\section{eQTL discovery}

eQTL analyses were carried out using FastQTL [18], using quantile normalized gene expression measures corrected for age, sex, RIN, sequencing batch, the first three genotype principal components (calculated in plink1.9, using LD pruned variants $\left(r^{2}>0.2\right.$ in $250 \mathrm{~kb}$ windows with a step size of $5 \mathrm{~kb}$ )), and 10 hidden confounders estimated through PEER [17]. FDR for each eQTL was calculated by first correcting $P$ values for the number of SNPs tested per gene/transcript (within $1 \mathrm{Mb}$ either side of the TSS) through estimation of a beta distribution using a minimum of 1000 permutations (maximum 10,000), and then correcting these $P$ values for the number of genes/transcripts tested using Storey's $q$ value method [30]. Details of all steps of these analyses are available at www.github.com/hobrien/GENEX-FB2.

\section{Comparisons with GTEx adult eQTL data}

We obtained multi-tissue meta-analysis results from the Genotype-Tissue Expression (GTEx) Consortium (V7) and matched variants by position and alleles. We 
determined which of our significant eGenes were assayed by GTEx from the median TPM results, and determine which genes were expressed in each tissue from the normalized expression matrices. Of the 1329 cis-eQTL eGenes (FDR < 0.05) identified in fetal brain, 176 were either not assayed (115 genes) or had expression values below the cut-off in all tissues (61 genes) in the GTEx study. For a further two fetal eGenes, there were no significant cis-eQTL that were genotyped by GTEx. For each eGene that was included in at least one GTEx expression matrix, we identified the most significant eQTL variant from our analysis that was assayed by GTEx and extracted the meta-analysis results and $P$ values for those eQTLs. For eQTLs that were detected in at least two GTEx tissues, we created a heatmap of $m$ values to show the extent of sharing between fetal brain and adult GTEx tissues. Tissue sharing was also quantified by estimating $\pi_{1}$ (the proportion of true positives [30]) among this set of eQTLs in each GTEx tissue.

\section{Spatiotemporal expression of fetal eGenes in human brain}

We used RNA sequencing data from dissected regions of the human pre- and post-natal brain, available through the BrainSpan project (http://www.brainspan.org/), to explore the regional and developmental expression of the fetal eGenes we identified. FPKM values were log transformed $\left(\log _{2}[\mathrm{FPKM}+0.0001]\right)$, clustered using Euclidian distances, and heatmaps were made using ggplot2 [66].

\section{eQTL enrichment analyses}

We performed the following enrichment analyses using GARFIELD [38]:

1) Enrichment of eQTL in functional annotation categories

2) Enrichment of mQTL in eQTL

3) Enrichment of variants associated with brain and non-brain complex traits in eQTL

Briefly, GARFIELD tests for enrichment of GWAS associated variants in annotation categories. The user provides the GWAS $P$ values for all variants and an annotation file indicating whether variants are located within functional categories to be tested. The software then selects an independent set of variants by sequentially removing variants with $r^{2}>0.1$ within a $1-\mathrm{Mb}$ window from the most significantly associated variant and then classifies each variant as overlapping a functional category if either the variant or a correlated variant (defined as $r^{2}>0.8$ ) is part of the annotation category. Statistical significance is calculated using a generalized linear model, while variants are matched by MAF, distance to the nearest transcription start site, number of LD proxies and $\mathrm{CpG}$ and $\mathrm{GC}$ content. $\mathrm{CpG}$ and GC content were calculated for a region 500 bp up and downstream of the variant using the BSgenome.Hsapiens.UCSC.hg19 $\mathrm{R}$ package. All variants from the UK10K were used as the background set of genetic variants. Depending on the particular enrichment analysis, our eQTL data were either considered as the GWAS trait (using the minimum $P$ value across all genes/transcripts tested) or the annotation, where all variants associated with at least one gene/transcript at 5\% FDR were used to construct the functional category.

\section{Enrichment of fetal brain eQTL in functional annotation categories}

We tested for significant enrichment of eQTLs in various functional and regulatory features defined using experimentally derived annotations from the ENCODE [8] and Epigenomics Roadmap [9] projects and provided with the GARFIELD software. Enrichment testing was performed for fetal brain transcript eQTLs with $P$ values equivalent to $\mathrm{FDR}<0.05$. We tested the default of all features in all assayed cells/tissues (1004 tests), defining $P$ values below the Bonferroni-corrected threshold of $P$ $<4.98 \times 10^{-5}$ as significant.

\section{Enrichment of fetal brain mQTLs in eQTL}

To test for enrichment of genetic variants associated with DNA methylation in the developing brain within fetal brain eQTL, we used a DNA methylation QTL (mQTL) dataset that we published previously using an overlapping set of fetal brain samples [10]. All FDR $<0.05 \mathrm{eQTLs}$ were used to define an annotation category. Enrichment was tested using the set of mQTL that we previously identified at a Bonferroni-corrected threshold $P<3.69 \times 10^{-13}$.

\section{Enrichment of variants associated with brain and non-brain complex traits in eQTLs}

GWAS results for attention deficit hyperactivity disorder [31], anorexia nervosa [32], autism spectrum disorder [33], bipolar disorder [34], major depressive disorder [35], neuroticism [26], schizophrenia [36], body mass index [39], coronary artery disease [40], inflammatory bowel disease [41], height [42], and type 2 diabetes [43] were downloaded, and the $P$ values for all variants were reformatted as input to GARFIELD. All 5\% FDR eQTLs for eTranscripts were used to define an annotation category. We tested for enrichment in fetal brain eQTLs of variants associated with each trait at multiple significance thresholds $\left(P<5 \times 10^{-5}, 5 \times 10^{-6}, 5 \times 10^{-7}, 5 \times\right.$ $10^{-8}$ ). In total, we tested 12 traits and 4 significance thresholds, highlighting enrichments that surpassed the Bonferroni corrected threshold $P<0.001$. 
Testing for pleiotropic association with neuropsychiatric traits We tested for joint associations between fetal brain eQTL and GWAS signals using summary-statistics-based Mendelian randomization (SMR) [44]. We tested all eGenes/ eTranscripts with eQTLs detected at FDR $<0.05$, using linkage disequilibrium information from the samples used for our eQTL analysis. GWAS minor allele frequencies were obtained from the 1000 Genomes Project (European populations). SMR $P$ values were Bonferroni corrected for the number of eGenes/eTranscripts tested. The HEIDI test was used to screen out joint associations that are likely to be driven by linkage using a nominal $P$ value threshold of 0.05 .

We additionally investigated potential sharing of eQTLs identified by the CommonMind Consortium in the adult human brain [49] that have been implicated in schizophrenia or neuroticism in the SMR study of Hauberg and colleagues [45]. For this, we restricted our analyses to the fetal brain eGenes detected at FDR $<0.05$ that overlapped with those implicated by Hauberg et al. in neuropsychiatric traits, and explored potential linkage disequilibrium between the top adult brain SMR eQTL and the most significant fetal brain eQTL for each gene. We report instances where the top adult brain SMR eQTL and the top eQTL for that gene in fetal brain have an $r^{2}>0.8$ in our samples, suggesting shared causal variants.

\section{Additional files}

Additional file 1: Tables S1-S10. containing sample data, top eQTL for all significant eGenes and eTranscripts (FDR $<0.05$ ), results of enrichment analyses and results of SMR analyses in relation to neuropsychiatric traits. (XLSX $987 \mathrm{~kb})$

Additional file 2: Figures S1-S8. All supplementary figures. (PDF $1212 \mathrm{~kb}$ )

\section{Acknowledgements}

We thank the Exeter Sequencing Service and Edinburgh Genomics for sequencing services. The human fetal material was provided by the Joint MRCNellcome Trust (grant \#099175/Z/12/Z) Human Developmental Biology Resource (www.hdbr.org). We thank iPSYCH and the ADHD, ASD, and Bipolar Disorder Working Groups of the Psychiatric Genomics Consortium for providing the summary statistics used in this study.

\section{Funding}

This work was supported by a Medical Research Council (UK) project grant to NJB (MR/L010674/2) and a Medical Research Council (UK) Centre grant to MJO (MR/L010305/1).

\section{Availability of data and materials}

The eQTL summary statistics data generated and analyzed in the current study are available in the figshare repository https://doi.org/10.6084/ m9.figshare.6881825 [57].

Individual RNA-Seq FASTQ files are available through the European Genomephenome Archive (https://ega-archive.org) under study accession EGAS00001003214 [67].

\section{Authors' contributions}

Primary analyses were performed by HEO'B and EH, with input from AFP and LSH. Laboratory work was performed by MJH, CCT, JEM, MJR, GM, and NJB. The study was designed by HEO'B, EH, CML, LCS, MJO, MCO'D, JM, and NJB.
HEO'B, EH, and NJB wrote the manuscript, with input from all other authors. All authors read and approved the final manuscript.

\section{Ethics approval and consent to participate}

This research was performed in accordance with the World Medical Association Declaration of Helsinki. Ethics approval for the collection and distribution of fetal material for scientific research was granted to the Human Developmental Biology Resource by the Royal Free Hospital research ethics committee (reference 08/H0712/34) and NRES Committee North East - Newcastle \& North Tyneside (reference 08/H0906/21+5). Consent for use of fetal material for medical research was provided by female donors. Additional ethics approval for genotyping and gene expression analyses of fetal samples was granted to NJB by the King's College London Psychiatry, Nursing and Midwifery Research Ethics Subcommittee (reference PNM/12/13-102).

\section{Consent for publication}

Consent for publication of research results in scientific journals was provided by female donors.

\section{Competing interests}

The authors declare that they have no competing interests.

\section{Publisher's Note}

Springer Nature remains neutral with regard to jurisdictional claims in published maps and institutional affiliations.

\section{Author details}

${ }^{1}$ MRC Centre for Neuropsychiatric Genetics \& Genomics, Division of Psychological Medicine \& Clinical Neurosciences, Cardiff University, Cardiff, UK. ${ }^{2}$ University of Exeter Medical School, University of Exeter, Exeter, UK. ${ }^{3}$ Institute of Psychiatry, Psychology \& Neuroscience, King's College London, London, UK. ${ }^{4}$ School of Biological Sciences, University of Essex, Colchester, UK. ${ }^{5}$ MRC Centre for Neuropsychiatric Genetics \& Genomics, Cardiff University School of Medicine, Hadyn Ellis Building, Maindy Road, Cardiff CF24 4HQ, UK.

Received: 27 May 2018 Accepted: 18 October 2018

Published online: 12 November 2018

\section{References}

1. Maurano MT, Humbert R, Rynes E, Thurman RE, Haugen E, Wang H, et al. Systematic localization of common disease-associated variation in regulatory DNA. Science. 2012;337:1190-5.

2. Barr CL, Misener VL. Decoding the non-coding genome: elucidating genetic risk outside the coding genome. Genes Brain Behav. 2016;15:187-204.

3. Stranger BE, Forrest MS, Dunning M, Ingle CE, Beazley C, Thorne N, et al. Relative impact of nucleotide and copy number variation on gene expression phenotypes. Science. 2007;315:848-53.

4. GTEx Consortium. Genetic effects on gene expression across human tissues. Nature. 2017;550:204-13.

5. Dimas AS, Deutsch S, Stranger BE, Montgomery SB, Borel C, Attar-Cohen $H_{\text {, }}$ et al. Common regulatory variation impacts gene expression in a cell typedependent manner. Science. 2009;325:1246-50.

6. Hill MJ, Bray NJ. Evidence that schizophrenia risk variation in the ZNF804A gene exerts its effects during fetal brain development. Am J Psychiatry. 2012;169:1301-8.

7. Hobert O. Gene regulation by transcription factors and microRNAs. Science. 2008;319:1785-6.

8. ENCODE Project Consortium. An integrated encyclopedia of DNA elements in the human genome. Nature. 2012;489:57-74.

9. Roadmap Epigenomics Consortium. Integrative analysis of 111 reference human epigenomes. Nature. 2015;518:317-30.

10. Hannon E, Spiers H, Viana J, Pidsley R, Burrage J, Murphy TM, et al. Methylation QTLs in the developing brain and their enrichment in schizophrenia risk loci. Nat Neurosci. 2016;19:48-54.

11. Weinberger DR. Implications of normal brain development for the pathogenesis of schizophrenia. Arch Gen Psychiatry. 1987;44:660-9.

12. Murray RM, Lewis SW. Is schizophrenia a neurodevelopmental disorder? Br Med J. 1987;295:681-2.

13. de la Torre-Ubieta L, Stein JL, Won H, Opland CK, Liang D, Lu D, et al. The dynamic landscape of open chromatin during human cortical neurogenesis. Cell. 2018;172:289-304. 
14. Tao R, Cousijn H, Jaffe AE, Burnet PW, Edwards F, Eastwood SL, et al. Expression of ZNF804A in human brain and alterations in schizophrenia, bipolar disorder, and major depressive disorder: a novel transcript fetally regulated by the psychosis risk variant rs1344706. JAMA Psychiatry. 2014;71: 1112-20.

15. Colantuoni C, Lipska BK, Ye T, Hyde TM, Tao R, Leek JT, et al. Temporal dynamics and genetic control of transcription in the human prefrontal cortex. Nature. 2011;478:519-23.

16. Haplotype Reference Consortium. A reference panel of 64,976 haplotypes for genotype imputation. Nat Genet. 2016;48:1279-83.

17. Stegle O, Parts L, Piipari M, Winn J, Durbin R. Using probabilistic estimation of expression residuals (PEER) to obtain increased power and interpretability of gene expression analyses. Nat Protocols. 2012;7:500-7.

18. Ongen H, Buil A, Brown AA, Dermitzakis ET, Delaneau O. Fast and efficient QTL mapper for thousands of molecular phenotypes. Bioinformatics. 2016; 32:1479-85.

19. Veyrieras JB, Kudaravalli S, Kim SY, Dermitzakis ET, Gilad Y, Stephens M, et al. High-resolution mapping of expression-QTLs yields insight into human gene regulation. PLoS Genet. 2008:4:e1000214

20. Gutierrez-Arcelus M, Lappalainen T, Montgomery SB, Buil A, Ongen H, et al. Passive and active DNA methylation and the interplay with genetic variation in gene regulation. Elife. 2013;2:e00523.

21. Wagner JR, Busche S, Ge B, Kwan T, Pastinen T, Blanchette M. The relationship between DNA methylation, genetic and expression inter-individual variation in untransformed human fibroblasts. Genome Biol. 2014;15:R37.

22. Stefansson H, Helgason A, Thorleifsson G, Steinthorsdottir V, Masson G, Barnard J, et al. A common inversion under selection in Europeans. Nat Genet. 2005;37:129-37.

23. Boettger LM, Handsaker RE, Zody MC, McCarroll SA. Structural haplotypes and recent evolution of the human 17q21.31 region. Nat Genet. 2012:44:881-5.

24. Smith DJ, Escott-Price V, Davies G, Bailey ME, Colodro-Conde L, Ward J, et al. Genome-wide analysis of over 106000 individuals identifies 9 neuroticismassociated loci. Mol Psychiatry. 2016;21:749-57.

25. Okbay A, Baselmans BM, De Neve JE, Turley P, Nivard MG, Fontana MA, et al. Genetic variants associated with subjective well-being, depressive symptoms, and neuroticism identified through genome-wide analyses. Nat Genet. 2016:48:624-33

26. Luciano M, Hagenaars SP, Davies G, Hill WD, Clarke TK, Shirali M, et al. Association analysis in over 329,000 individuals identifies 116 independent variants influencing neuroticism. Nat Genet. 2018;50:6-11.

27. Kouri N, Ross OA, Dombroski B, Younkin CS, Serie DJ, Soto-Ortolaza A, et al. Genome-wide association study of corticobasal degeneration identifies risk variants shared with progressive supranuclear palsy. Nat Commun. 2015;6:7247.

28. Desikan RS, Schork AJ, Wang Y, Witoelar A, Sharma M, McEvoy LK, et al. Genetic overlap between Alzheimer's disease and Parkinson's disease at the MAPT locus. Mol Psychiatry. 2015;20:1588-95.

29. Simón-Sánchez J, Schulte C, Bras JM, Sharma M, Gibbs JR, Berg D, et al. Genome-wide association study reveals genetic risk underlying Parkinson's disease. Nat Genet. 2009;41:1308-12.

30. Storey JD, Tibshirani R. Statistical significance for genomewide studies. Proc Natl Acad Sci. 2003;100:9440-5.

31. Demontis D, Walters RK, Martin J, Mattheisen M, Damm Als T, Agerbo E, et al. Discovery of the first genome-wide significant risk loci for ADHD. Preprint at. https://www.biorxiv.org/content/early/2017/06/03/145581.

32. Duncan L, Yilmaz Z, Gaspar H, Walters R, Goldstein J, Anttila V, et al. Significant locus and metabolic genetic correlations revealed in genome-wide association study of anorexia nervosa. Am J Psychiatry. 2017;174:850-8.

33. Grove J, Ripke S, Damm Als T, Mattheisen M, Walters R, Won H, et al. Common risk variants identified in autism spectrum disorder. Preprint at: https://www.biorxiv.org/content/early/2017/11/27/224774

34. Stahl E, Breen G, Forstner A, McQuillin A, Ripke S, Bipolar disorder working Group of the Psychiatric Genomics Consortium. Genomewide association study identifies 30 loci associated with bipolar disorder. Preprint at: https:// www.biorxiv.org/content/early/2018/01/24/173062

35. Wray NR, Ripke S, Mattheisen M, Trzaskowski M, Byrne EM, Abdellaoui A, et al. Genome-wide association analyses identify 44 risk variants and refine the genetic architecture of major depression. Nat Genet. 2018;50:668-81.

36. Pardiñas AF, Holmans P, Pocklington AJ, Escott-Price V, Ripke S, Carrera N, et al. Common schizophrenia alleles are enriched in mutation-intolerant genes and in regions under strong background selection. Nat Genet. 2018; 50:381-9.
37. Takata A, Matsumoto N, Kato T. Genome-wide identification of splicing QTLs in the human brain and their enrichment among schizophrenia-associated loci. Nat Commun. 2017;8:14519.

38. lotchkova V, Ritchie GRS, Geihs M, Morganella S, Min, JL, Walter K, et al. GARFIELD - GWAS analysis of regulatory or functional information enrichment with LD correction. Preprint at: https://www.biorxiv.org/ content/early/2016/11/07/085738

39. Locke AE, Kahali B, Berndt SI, Justice AE, Pers TH, Day FR, et al. Genetic studies of body mass index yield new insights for obesity biology. Nature. 2015;518:197-206

40. CARDIoGRAMplusC4D Consortium. A comprehensive 1,000 Genomes-based genome-wide association meta-analysis of coronary artery disease. Nat Genet. 2015:47:1121-30.

41. Liu JZ, van Sommeren S, Huang H, Ng SC, Alberts R, Takahashi A, et al. Association analyses identify 38 susceptibility loci for inflammatory bowel disease and highlight shared genetic risk across populations. Nat Genet. 2015:47:979-86

42. Wood AR, Esko T, Yang J, Vedantam S, Pers TH, Gustafsson S, et al. Defining the role of common variation in the genomic and biological architecture of adult human height. Nat Genet. 2014;46:1173-86.

43. Morris AP, Voight BF, Teslovich TM, Ferreira T, Segrè AV, Steinthorsdottir V, et al. Large-scale association analysis provides insights into the genetic architecture and pathophysiology of type 2 diabetes. Nat Genet. 2012;44: 981-90.

44. Zhu Z, Zhang F, Hu H, Bakshi A, Robinson MR, Powell JE, et al. Integration of summary data from GWAS and eQTL studies predicts complex trait gene targets. Nat Genet. 2016;48:481-7.

45. Hauberg ME, Zhang W, Giambartolomei C, Franzén O, Morris DL, Vyse TJ, et al. Large-scale identification of common trait and disease variants affecting gene expression. Am J Hum Genet. 2017;100:885-94.

46. Hannon E, Weedon M, Bray N, O'Donovan M, Mill J. Pleiotropic effects of trait-associated genetic variation on DNA methylation: utility for refining GWAS loci. Am J Hum Genet. 2017;100:954-9.

47. Gusev A, Mancuso N, Won H, Kousi M, Finucane HK, Reshef $Y$, et al. Transcriptome-wide association study of schizophrenia and chromatin activity yields mechanistic disease insights. Nat Genet. 2018;50:538-48.

48. Sekar A, Bialas AR, de Rivera H, Davis A, Hammond TR, Kamitaki N, et al. Schizophrenia risk from complex variation of complement component 4. Nature. 2016;530:177-83.

49. Fromer M, Roussos P, Sieberts SK, Johnson JS, Kavanagh DH, Perumal TM, et al. Gene expression elucidates functional impact of polygenic risk for schizophrenia. Nat Neurosci. 2016;19:1442-53.

50. Bray NJ, Buckland PR, Owen MJ, O'Donovan MC. Cis-acting variation in the expression of a high proportion of genes in human brain. Hum Genet. 2003;113:149-53.

51. Ge B, Pokholok DK, Kwan T, Grundberg E, Morcos L, Verlaan DJ, et al. Global patterns of cis variation in human cells revealed by high-density allelic expression analysis. Nat Genet. 2009;41:1216-22.

52. de Jong $S$, Chepelev I, Janson E, Strengman E, van den Berg LH, Veldink JH, et al. Common inversion polymorphism at 17q21.31 affects expression of multiple genes in tissue-specific manner. BMC Genomics. 2012;13:458.

53. de Wit J, Ghosh A. Control of neural circuit formation by leucine-rich repeat proteins. Trends Neurosci. 2014;37:539-50.

54. Swanwick CC, Shapiro ME, Vicini S, Wenthold RJ. Flotillin-1 promotes formation of glutamatergic synapses in hippocampal neurons. Dev Neurobiol. 2010;70:875-83.

55. Harrison PJ. The hippocampus in schizophrenia: a review of the neuropathological evidence and its pathophysiological implications. Psychopharmacology. 2004;174:151-62.

56. Tau GZ, Peterson BS. Normal development of brain circuits. Neuropsychopharmacology. 2010;35:147-68.

57. O'Brien HE, Bray NJ. Summary statistics for expression quantitative trait loci in the developing human brain and their enrichment in neuropsychiatric disorders. 2018. figshare. Fileset. https://doi.org/10.6084/m9.figshare.6881825.

58. Zhao H, Sun Z, Wang J, Huang H, Kocher JP, Wang L. CrossMap: a versatile tool for coordinate conversion between genome assemblies. Bioinformatics. 2014;30:1006-7.

59. Wang L, Wang S, Li W. RSeQC: quality control of RNA-seq experiments. Bioinformatics. 2012;28:2184-5.

60. Kim D, Langmead B, Salzberg SL. HISAT: a fast spliced aligner with low memory requirements. Nat Methods. 2015;12:357-60. 
61. Bray NL, Pimentel H, Melsted P, Pachter L. Near-optimal probabilistic RNAseq quantification. Nat Biotechnol. 2016;34:525-7.

62. Soneson C, Love MI, Robinson MD. Differential analyses for RNA-seq transcript-level estimates improve gene-level inferences. F1000Research. 2015;4:1521.

63. Durinck S, Moreau Y, Kasprzyk A, Davis S, De Moor B, Brazma A, et al. BioMart and Bioconductor: a powerful link between biological databases and microarray data analysis. Bioinformatics. 2005;21:3439-40.

64. Love Ml, Huber W, Anders S. Moderated estimation of fold change and dispersion for RNA-seq data with DESeq2. Genome Biol. 2014;15:550.

65. Hoffman GE, Schadt EE. variancePartition: interpreting drivers of variation in complex gene expression studies. BMC Bioinformatics. 2016;17:483.

66. Wickham H. ggplot22: elegant graphics for data analysis. 2nd ed. New York: Springer; 2016.

67. Bray N. FBSeq: RNA sequencing of human fetal brain. European Genomephenome Archive. 2018; https://ega-archive.org/studies/EGAS00001003214

68. Sul JH, Han B, Ye C, Choi T, Eskin E. Effectively identifying eQTLs from multiple tissues by combining mixed model and meta-analytic approaches. PLoS Genet. 2013;9:e1003491

Ready to submit your research? Choose BMC and benefit from:

- fast, convenient online submission

- thorough peer review by experienced researchers in your field

- rapid publication on acceptance

- support for research data, including large and complex data types

- gold Open Access which fosters wider collaboration and increased citations

- maximum visibility for your research: over $100 \mathrm{M}$ website views per year

At $\mathrm{BMC}$, research is always in progress.

Learn more biomedcentral.com/submissions 